\title{
Avaliação médico-pericial da capacidade laborativa dos portadores de serosite lúpica
}

\author{
Medical expert evaluation of work capacity of \\ patients with lupus serositis
}

Nancy Segalla Rosa Chammas'; Raquel Barbosa Cintra ${ }^{2}$

\begin{abstract}
Chammas NSR; Cintra RB. Avaliação médico-pericial da capacidade laborativa dos portadores de serosite lúpica. Saúde, Ética \& Justiça. 2014;19(1);12-20.

RESUMO: Lúpus eritematoso sistêmico (LES) é uma doença inflamatória crônica de caráter autoimune, de etiologia não esclarecida, que acomete predominantemente o sexo feminino na frequência de 9:1, podendo atingir diversos órgãos e sistemas, sendo mais comumente pele, articulações e rins. Entretanto, a serosite lúpica apresenta incidência significativa, ao redor de $50 \%$ dos casos, com comprometimento pericárdico e pleural, cujas manifestações clínicas podem determinar incapacidade laborativa. $\mathrm{O}$ objetivo deste trabalho foi distinguir quando a serosite lúpica leva à incapacidade, resultando na concessão de benefício previdenciário. Para tanto, foi feita uma revisão de literatura sobre o tema, visando a avaliação da capacidade para o trabalho de portadores de serosite lúpica. A atividade lúpica pode ser capaz de causar incapacidade para o trabalho. A maioria das pessoas com LES pode ter uma vida produtiva normal; no entanto, alguns pacientes têm uma evolução mais complicada e perdem a capacidade produtiva. Alguns sinais e sintomas da serosite lúpica podem mostrar a incapacidade. É possível concluir que a incapacidade decorrente da doença ativa é em geral total e temporária, exigindo reavaliações periódicas com o objetivo de verificação clínico-laboratorial da evolução da doença.
\end{abstract}

DESCRITORES: Serosite; Lúpus eritematoso sistêmico; Avaliação da capacidade de trabalho.

1. Juizado Especial Federal de São Paulo/SP - JEF/SP; Instituto de Medicina Social e de Criminologia de São Paulo - IMESC.

2. Universidade de Moji das Cruzes - UMC.

Endereço para correspondência: Avenida Paulista, 1345, $1^{\circ}$ Subsolo, CEP 01311-200. E-mail: nancysegalla@hotmail.com. 


\section{Introdução}

$\mathrm{O}$ lúpus eritematoso sistêmico (LES) é uma doença de curso crônico, na qual há atividade inflamatória de caráter imunológico com produção de autoanticorpos. Sua etiologia não foi estabelecida, porém pode estar associada a fatores genéticos, hormonais e ambientais. Pode atingir qualquer tecido ou órgão e, frequentemente, cursa com serosite. A doença é crônica, incurável, evoluindo com períodos de exacerbação e períodos de remissão ou oligossintomáticos ${ }^{1}$. O LES encontra-se na Classificação Internacional das Doenças, $10^{\text {a }}$ edição (CID-10), no capítulo sobre Doenças do sistema osteomuscular e do tecido conjuntivo, sendo codificado como $\mathrm{M} 32^{2}$.

LES é uma doença rara, e, segundo estudo norteamericano $^{1}$, sua frequência é de 14 a 50 por 100.000 pessoas. Ocorre nove vezes mais em mulheres do que em homens, sendo que normalmente se inicia na fase reprodutiva. Pode ocorrer em todas as raças e em todas as partes do mundo ${ }^{1}$. A evolução da doença para óbito pode estar associada à atividade da doença, a complicações decorrentes do tratamento ou a outras causas não diretamente relacionadas à patologia. O prognóstico dos pacientes com LES tem melhorado nos últimos anos, condição atribuída a vários fatores como diagnóstico precoce, introdução de novos medicamentos e controle das infecções recorrentes ${ }^{3}$.

O LES apresenta diversas manifestações clínicas, sendo frequente a serosite pleural e pericárdica. A serosite pode ser encontrada em aproximadamente $50 \%$ dos casos em necropsia de portadores da doença, indicando sua elevada frequência ${ }^{4}$. As manifestações pulmonares incluem dor torácica ventilatório-dependente, dispneia, redução do murmúrio vesicular na ausculta, derrame pleural sugerido pela macicez à percussão, confirmado em exame radiológico. O quadro cardíaco inclui dor precordial, atrito pericárdico ou abafamento de bulhas, e o derrame pericárdico pode ser confirmado por ecocardiograma. Em menos de 1\% dos casos, há evolução para tamponamento cardíaco ${ }^{4}$.

Dados do Instituto Nacional de Seguro Social (INSS) revelam que o LES tem gerado auxílios-doença e aposentadorias por invalidez ${ }^{5}$. De acordo com os dados do anuário estatístico do INSS, o LES foi responsável por 72.092 aposentadorias por invalidez entre 2009 e 2011, aproximadamente $20 \%$ das aposentadorias por invalidez do período, e pela concessão de 937.087 benefícios de auxílio-doença no mesmo período, correspondendo a cerca de $20 \%$, conforme mostram as tabelas 1,2 e $3^{5}$.

TABELA 1 - Número de benefícios concedidos pela Previdência Social no total e em casos de Lúpus Eritematoso Sistêmico

\begin{tabular}{|c|c|c|c|c|c|}
\hline \multirow{2}{*}{ Ano } & \multirow{2}{*}{$\begin{array}{l}\text { Total de benefícios } \\
\text { da Previdência }\end{array}$} & \multicolumn{2}{|c|}{ Aposentadoria por invalidez } & \multicolumn{2}{|c|}{ Auxílio-doença } \\
\hline & & Total & LES & Total & LES \\
\hline 2009 & 3.389 .219 & 158.285 & $25.051(15,8 \%)$ & 1.525 .083 & $295.883(19,4 \%)$ \\
\hline 2010 & 3.565 .641 & 160.624 & $23.556(14,7 \%)$ & 1.686 .759 & $327.238(19,4 \%)$ \\
\hline 2011 & 3.737 .177 & 159.377 & $23.485(14,7 \%)$ & 1.814 .257 & $350.016(19,3 \%)$ \\
\hline
\end{tabular}

Fonte: Anuário estatístico do INSS, $2012^{5}$

TABELA 2 - Aposentadorias por invalidez concedidas por sexo, de 2009 a 2011

\begin{tabular}{|c|c|c|c|c|c|c|}
\hline \multirow{4}{*}{ Ano } & \multicolumn{6}{|c|}{ APOSENTADORIAS URBANAS POR INVALIDEZ CONCEDIDAS } \\
\hline & \multicolumn{3}{|c|}{ Quantidade } & \multicolumn{3}{|c|}{ Valor (R\$ mil) } \\
\hline & \multirow{2}{*}{ Total } & \multicolumn{2}{|c|}{ Sexo } & \multirow{2}{*}{ Total } & \multicolumn{2}{|c|}{ Sexo } \\
\hline & & Masculino & Feminino & & Masculino & Feminino \\
\hline 2009 & 25.051 & $\begin{array}{c}14.209 \\
(56,7 \%)\end{array}$ & $\begin{array}{c}10.842 \\
(43,3 \%)\end{array}$ & 22.616 & 15.294 & 7.322 \\
\hline 2010 & 23.556 & $\begin{array}{c}13.235 \\
(56,2 \%)\end{array}$ & $\begin{array}{c}10.321 \\
(43,9 \%)\end{array}$ & 23.057 & 15.537 & 7.520 \\
\hline 2011 & 23.485 & $\begin{array}{c}12.866 \\
(54,8 \%)\end{array}$ & $\begin{array}{c}10.619 \\
(45,2 \%)\end{array}$ & 24.073 & 15.841 & 8.232 \\
\hline
\end{tabular}


TABELA 3 - Auxílios-doença concedidos por sexo, de 2009 a 2011

\begin{tabular}{|c|c|c|c|c|c|c|}
\hline \multirow{4}{*}{ Ano } & \multicolumn{6}{|c|}{ AUXÍLIOS-DOENÇA CONCEDIDOS } \\
\hline & \multicolumn{3}{|c|}{ Quantidade } & \multicolumn{3}{|c|}{ Valor (R\$ mil) } \\
\hline & \multirow{2}{*}{ Total } & \multicolumn{2}{|c|}{ Sexo } & \multirow{2}{*}{ Total } & \multicolumn{2}{|c|}{ Sexo } \\
\hline & & Masculino & Feminino & & Masculino & Feminino \\
\hline 2009 & 295.833 & $\begin{array}{l}150.761 \\
(51,0 \%)\end{array}$ & $\begin{array}{c}145.072 \\
(49,0 \%)\end{array}$ & 28.747 & 15.636 & 13. 111 \\
\hline 2010 & 327.238 & $\begin{array}{l}162.483 \\
(49,6 \%)\end{array}$ & $\begin{array}{l}164.755 \\
(50,4 \%)\end{array}$ & 32.520 & 17.728 & 14. 792 \\
\hline 2011 & 350.016 & $\begin{array}{l}173.310 \\
(49,5 \%)\end{array}$ & $\begin{array}{l}176.706 \\
(50,5 \%)\end{array}$ & 31.794 & 17. 381 & 14. 413 \\
\hline
\end{tabular}

Fonte: Anuário estatístico do INSS, $2012^{5}$

O LES é responsável por centenas de benefícios previdenciários, desta forma entende-se que estudos sobre a serosite lúpica podem ser úteis ao médico perito previdenciário durante a avaliação de incapacidade laborativa.

\section{Objetivos}

\section{Objetivo geral}

O objetivo do trabalho é citar quais achados de exame físico podem ser utilizados na avaliação médicopericial previdenciária dos portadores de LES com serosite lúpica e sua repercussão funcional frente à avaliação da capacidade laborativa.

\section{Objetivo específico}

Pretende-se distinguir quais manifestações clínicas da serosite lúpica podem levar à incapacidade, resultando na concessão de benefício previdenciário.

\section{Métodos}

Foi realizada revisão de literatura com os descritores: "lúpus eritematoso sistêmico", "serosite", "pericardite", "pleurisia" e seus respectivos em inglês. Os descritores foram utilizados de forma isolada e combinada. As bases de dados consultadas foram Pub Med e Scielo. Foram excluídos artigos publicados em língua diversa de inglês e/ou português e artigos que não estavam disponíveis gratuitamente online. Foram encontrados 497 de artigos, e, após aplicados os critérios de exclusão, restaram 12 artigos publicados entre os anos 1988 e 2013.

\section{Resultados}

A atividade do LES cursa com sintomas que incluem febre, fadiga, emagrecimento, edema, alopecia, úlceras mucosas, vasculite dérmica, fenômeno de Raynaud, telangiectasias, eritema multiforme, poliartrite, nefrite, acometimento neurológico e psiquiátrico, manifestações digestivas, hepáticas, hematológicas, cardiovasculares, pleuropulmonares, oculares, entre outros, indicando a necessidade de uma visão global da doença ${ }^{6}$. De acordo com os critérios de classificação do LES do American College of Rheumatology, revisados em 1997, a serosite consta como um dos onze critérios que definem o diagnóstico: serosite pleurite (caracterizada por história convincente de dor pleurítica, atrito auscultado pelo médico ou evidência de derrame pleural) ou pericardite (documentado por eletrocardiograma, atrito ou evidência de derrame pericárdico $)^{1}$.

Em 50\% dos pacientes, ocorre o envolvimento pleural ou pulmonar, com pleurite e derrame pleural geralmente bilateral. Pode cursar com hipertensão pulmonar em 12 a $23 \%$ dos casos, de intensidade leve a moderada ${ }^{7}$. A inflamação pulmonar aguda cursa com dispneia, tosse, febre, hemoptise, pleurisia, com exame radiológico do tórax mostrando infiltrado alveolar em bases, com derrame pleural em $50 \%$ dos casos. O derrame pleural causa dor torácica variável com os 
movimentos respiratórios, dispneia e atrito pleural, com exame radiológico mostrando volumoso derrame pleural que pode ser bilateral ${ }^{8}$.

A pericardite ocorre comumente no LES, em aproximadamente $55 \%$ dos pacientes, podendo ser clínica ou subclínica, geralmente com discreto derrame pericárdico que raramente evolui para pericardite constritiva ou tamponamento cardíaco. Em 25\% dos casos, a pericardite pode estar associada à miocardite, com espessamento valvar ${ }^{7}$. A pericardite cursa com dor torácica, atrito pericárdico, abafamento de bulhas, pulso paradoxal, estase jugular, com exame radiológico do tórax mostrando aumento da área cardíaca. O ecocardiograma evidencia espessamento pericárdico e derrame pericárdico ${ }^{8}$. Em estudo feito por Alves ${ }^{9}$, com avaliação realizada em dois grupos de portadores do LES, um fora de atividade e outro em atividade, mostrou que o acometimento pericárdico ocorreu em 31,2\% dos casos dos pacientes em atividade e foi inexpressivo nos pacientes fora de atividade lúpica. $\mathrm{O}$ acometimento pericárdico foi caracterizado por derrame detectável ao ecocardiograma e a pericardite seca diagnosticada pelo quadro clínico e eletrocardiograma, mostrando lesões mínimas não detectáveis ao ecocardiograma. Derrames pericárdicos discretos são comuns no LES, e a ocorrência de grande derrame pericárdico pode ser a primeira manifestação da doença. Os pacientes com lúpus fora de atividade não mostraram alterações pericárdicas, indicando que estas alterações se associam à fase aguda do LES 9 .

No tratamento do LES em fase de exacerbação, medidas gerais devem ser tomadas pelo médico assistente, como orientação do paciente e familiares quanto à doença, indicação de repouso durante o período de atividade inflamatória, evitar o tabagismo, controle dos fatores de risco cardiovasculares como hipertensão arterial sistêmica, diabetes, dislipidemia, dieta balanceada evitando excesso de sal e gorduras ${ }^{1}$. É importante que o médico perito saiba que em períodos de exacerbação, o portador de LES pode ser orientado a fazer repouso.

Consideram-se como manifestações leves do LES quadros em que a doença está clinicamente estável, sem comprometimento de funções vitais e ausência de toxicidade do tratamento medicamentoso. O LES grave é definido quando há mau prognóstico a curto ou longo prazo, ou já houver comprometimento de um ou mais órgãos, podendo evoluir para danos irreversíveis, comprometimento funcional ou diminuição da sobrevida, incluindo pericardite com tamponamento $\operatorname{cardíaco}^{10}$.

Critérios de hospitalização incluem os quadros de risco moderado a alto para complicações e necessidade de terapêutica intra-hospitalar, que incluem os quadros de derrame pleural com dispneia e derrame pericárdico com síndrome de restrição diastólica ${ }^{10}$.

O tratamento do LES inclui várias classes medicamentosas, podendo associar medicamentos de acordo com as manifestações clínicas e órgãos acometidos. De forma geral, os anti-inflamatórios hormonais e imunossupressores são largamente utilizados. A serosite cardíaca e pulmonar geralmente apresenta boa resposta ao tratamento medicamentoso com corticosteróides em doses moderadas ${ }^{11}$. Sendo assim, o médico perito deve verificar se o periciando está em tratamento medicamentoso.

É importante destacar que não há um período estabelecido para a duração do tratamento. Após atingir a remissão clínica, as doses dos medicamentos podem ser diminuídas gradualmente, em intervalos mensais, ao longo de aproximadamente seis a doze meses, sob monitorização laboratorial sistemática da atividade da doença. $\mathrm{O}$ tratamento medicamentoso visa ao controle da atividade da doença, controle e impedimento do surgimento de fatores de risco para complicações crônicas do $\mathrm{LES}^{7}$.

A atividade do LES pode ser objetivamente avaliada através do Systemic Lupus Erythematosus Disease Activity Index - SLEDAI ${ }^{3}$, exposto no quadro 1 , composto de 24 itens entre sinais, sintomas e exames laboratoriais, cada um recebendo um peso que varia de 1 a 8 , correspondendo à gravidade da manifestação. Quanto mais elevado o escore final, maior é a gravidade da atividade da doença ${ }^{12}$.

O LES pode evoluir com lesões permanentes ou sequelas, em decorrência da própria doença como insuficiência renal crônica, pneumonite com fibrose pulmonar, ou em função do tratamento instituído, como os efeitos secundários dos corticosteróides como osteoporose, osteonecrose, hipertensão arterial sistêmica, aterosclerose; os efeitos dos imunossupressores no surgimento de infecções, infertilidade, surgimento tardio de neoplasias. A cloroquina pode causar maculopatia por impregnação na retina, podendo resultar em alteração da visão. Desta forma, o LES e o tratamento constituem significativas causas de morbimortalidade. Para avaliação da repercussão dos danos durante a evolução do LES, pode ser utilizado o índice Systemic Lupus International Collaborating Clinics / American College of Rheumatology - SLICC/ACR/DI, mostrado no quadro 2. Este índice avalia doze itens de danos irreversíveis, incluindo os resultantes da doença, terapêutica e comorbidades, considerando as lesões presentes e irreversíveis há no mínimo seis meses, não decorrentes de inflamação ativa. No decorrer do acompanhamento, o índice de dano pode permanecer estável ou aumentar, com pontuação máxima de 47 $\operatorname{pontos}^{12,13}$. 
Chammas NSR; Cintra RB. Avaliação médico-pericial da capacidade laborativa dos portadores de serosite lúpica.

QUADRO 1 - Índice de atividade do Lúpus Eritematoso Sistêmico [Systemic Lupus Erythematosus Disease Activity Index (SLEDAI)]

\begin{tabular}{|c|c|}
\hline Escore & Item \\
\hline 8 & $\begin{array}{l}\text { Convulsão - início recente, excluindo outras causas, tais como distúrbios metabólicos, infecções ou } \\
\text { medicamentos. }\end{array}$ \\
\hline 8 & $\begin{array}{l}\text { Psicose - distúrbio na percepção da realidade, incluindo alucinações, delírios, incoerências, perda de } \\
\text { associações, pensamento não lógico, comportamento bizarro, desorganizado ou catatônico. Excluir outras } \\
\text { causas, tais como uremia ou medicações. }\end{array}$ \\
\hline 8 & $\begin{array}{l}\text { Síndrome cerebral orgânica - alteração da função mental, com prejuízo na orientação, memória ou outras } \\
\text { funções intelectuais, com rápido surgimento e flutuações, incapacidade de sustentar a atenção, somado } \\
\text { a pelo menos dois dos que seguem: distúrbio da percepção, diálogo incoerente, insônia, sonolência e } \\
\text { aumento ou diminuição da atividade psicomotora. Excluir outras causas: distúrbios metabólicos, infecções } \\
\text { ou medicações. }\end{array}$ \\
\hline 8 & $\begin{array}{l}\text { Visual - Alterações no fundo do olho, tais como corpos citóides, hemorragias retinianas, exsudatos } \\
\text { ou hemorragias na coróide ou nervo óptico. Excluir outras causas tais como hipertensão, infecções ou } \\
\text { medicamentos. }\end{array}$ \\
\hline 8 & Nervos cranianos - Surgimento de neuropatia sensitiva ou motora nos nervos cranianos. \\
\hline 8 & Cefaleia lúpica - Persistente e grave, pode ser enxaquecosa, com pouca resposta a analgésicos opióides. \\
\hline 8 & AVC - evento de início recente e não relacionado com aterosclerose ou hipertensão. \\
\hline 8 & $\begin{array}{l}\text { Vasculite - Ulceração, gangrena, nódulo, infarto periungueal, hemorragias puntiformes, biópsia ou } \\
\text { arteriografia compatível com vasculite. }\end{array}$ \\
\hline 4 & Artrite - duas articulações ou mais com sinais flogísticos. \\
\hline 4 & $\begin{array}{l}\text { Miosite-Fraqueza ou dor muscular proximal com elevação de creatinofosfoquinase ou aldolase, ou } \\
\text { eletroneuromiografia compatível com miosite ou biópsia com infiltrado inflamatório em fibra muscular. }\end{array}$ \\
\hline 4 & Cilindros - Hemáticos ou granulosos. \\
\hline 4 & Hematúria - Mais de 5 hemácias/campo de grande aumento. Excluir cálculos, infecções ou outras causas. \\
\hline 4 & Proteinúria - acima de $0,5 \mathrm{~g} / 24 \mathrm{~h}$. \\
\hline 4 & Piúria - Mais de 5 leucócitos/campo de grande aumento. Excluir infecção. \\
\hline 2 & Rash malar novo. \\
\hline 2 & Alopecia - Perda de cabelo anormal, difusa ou localizada. \\
\hline 2 & Membranas mucosas - Ulcerações nasais ou orais. \\
\hline 2 & Pleurite - Dor pleurítica com atrito pleural, ou derrame pleural ou espessamento pleural. \\
\hline 2 & $\begin{array}{l}\text { Pericardite - dor compatível com pericardite somada a pelo menos um dos seguintes achados: atrito } \\
\text { pericárdico, derrame pericárdico, eletrocardiograma ou ecocardiograma compatíveis com pericardite. }\end{array}$ \\
\hline 2 & $\begin{array}{l}\text { Baixos complementos - Diminuição do } \mathrm{CH} 50, \mathrm{C} 3 \text { ou C4 abaixo do limite de normalidade, de acordo com } \\
\text { os valores de referência do exame. }\end{array}$ \\
\hline 1 & Febre - Acima de $38^{\circ}$, excluindo infecções. \\
\hline 1 & Trombocitopenia - Menos de 100.000 plaquetas $/ \mathrm{mm}^{3}$, excluindo outras causas, tais como medicamentos. \\
\hline 1 & Leucopenia - Menos de 3000 leucócitos $/ \mathrm{mm}^{3}$, excluindo outras causas, tais como medicamentos. \\
\hline To & \\
\hline
\end{tabular}

Fonte: Freire, $2011^{12}$. 
QUADRO 2 - Morbidades cumulativas do Lúpus Eritematoso Sistêmico [Systemic Lupus International Collaborating Clinics/ American College of Rheumatology (SLICC/ACR/DI)]

\begin{tabular}{|c|c|}
\hline Item & Escore \\
\hline $\begin{array}{l}\text { Alterações oculares } \\
\text { - Nunca teve catarata } \\
\text { - Alterações na retina ou atrofia do nervo óptico }\end{array}$ & $\begin{array}{l}1 \\
1\end{array}$ \\
\hline $\begin{array}{l}\text { Alterações Neuropsiquiátricas } \\
\text { - Alteração de qualquer grau cognitivo } \\
\text { - Infarto que requeira terapia por } 6 \text { meses } \\
\text { - AVE anterior (se mais de } 1 \text { vez: score 2) } \\
\text { - Neuropatia (excluindo óptica) } \\
\text { - Mielite transversa }\end{array}$ & $\begin{array}{l}1 \\
1 \\
1(2) \\
1 \\
1\end{array}$ \\
\hline $\begin{array}{l}\text { Alterações renais } \\
\text { - Taxa de filtração glomerular }<50 \% \\
\text { - Doença renal terminal } \\
\text { - Proteinúria }>3,5 \mathrm{~g} / \text { dia }\end{array}$ & $\begin{array}{l}1 \\
3 \\
1\end{array}$ \\
\hline $\begin{array}{l}\text { Alterações Pulmonares } \\
\text { - Hipertensão Pulmonar } \\
\text { - Fibrose Pulmonar } \\
\text { - Pulmão Evanescente (radiografia) } \\
\text { - Fibrose Pleural (radiografia) } \\
\text { - Infarto Pulmonar (radiografia) }\end{array}$ & $\begin{array}{l}1 \\
1 \\
1 \\
1 \\
1\end{array}$ \\
\hline $\begin{array}{l}\text { Alterações Cardiovasculares } \\
\text { - Angina ou bypass } \\
\text { - Infarto agudo do miocárdio anterior (score } 2 \text { se mais de } 2 \text { vezes) } \\
\text { - Disfunção Ventricular } \\
\text { - Doença valvular } \\
\text { - Pericardite por } 6 \mathrm{~m} \text { ou pericardiotomia }\end{array}$ & $\begin{array}{c}1 \\
1(2) \\
1 \\
1 \\
1\end{array}$ \\
\hline $\begin{array}{l}\text { Alteração Vascular Periférica } \\
\text { - Claudicação por } 6 \mathrm{~m} \\
\text { - Perda de tecido irrisória } \\
\text { - Perda significante de tecido ( } 1 \text { dedo)- score maior (2) quando em mais de uma localização } \\
\text { - Trombose venosa com ulceração, etc. }\end{array}$ & $\begin{array}{c}1 \\
1 \\
1(2) \\
1\end{array}$ \\
\hline $\begin{array}{l}\text { Alterações do trato gastrointestinal (TGI) } \\
\text { - Infarto ou ressecção de parte do intestino (score vai para } 2 \text { quando em mais de } 1 \text { local) } \\
\text { - Insuficiência Mesentérica } \\
\text { - Peritonite crônica } \\
\text { - Ter feito alguma vez manipulação do TGI ou cirurgia }\end{array}$ & $\begin{array}{c}1(2) \\
1 \\
1 \\
1\end{array}$ \\
\hline $\begin{array}{l}\text { Alterações do Aparelho Musculoesquelético } \\
\text { - Atrofia muscular ou fraqueza } \\
\text { - Artrite deformante ou erosiva (inclui Jaccoud e exclui necrose avascular) } \\
\text { - Osteoporose c/ fratura ou colapso vertebral (exclui necrose avascular) } \\
\text { - Necrose Avascular (score 2, se em mais de } 1 \text { local) } \\
\text { - Osteomielite }\end{array}$ & $\begin{array}{c}1 \\
1 \\
1 \\
1(2) \\
1\end{array}$ \\
\hline $\begin{array}{l}\text { Alterações Mucocutâneas } \\
\text { - Alopecia crônica } \\
\text { - Paniculite } \\
\text { - Úlceras por 6meses (excluir trombose) }\end{array}$ & $\begin{array}{l}1 \\
1 \\
1\end{array}$ \\
\hline Falência gonadal precoce & 1 \\
\hline Diabetes pelo tratamento & 1 \\
\hline Doença maligna, excluída displasia cervical (score 2 se em mais de 1 local) & $1(2)$ \\
\hline
\end{tabular}

Observação: As alterações irreversíveis relacionadas ao LES ou ao tratamento, mantidos por seis meses desde o início da doença, sem outra causa.

Fonte: Guarize, $2004^{13}$. 


\section{Discussão}

A maioria das pessoas com LES pode ter uma vida produtiva normal. No entanto, alguns pacientes têm uma evolução mais complicada e perdem a capacidade produtiva. Nos casos em que há perda da capacidade laborativa, caso o doente seja beneficiário do INSS, ele pode receber algum benefício da previdência social.

Entende-se por incapacidade laborativa a impossibilidade de desempenho do servidor de atividades específicas para o seu cargo, função ou emprego, decorrentes das alterações patológicas consequentes aos agravos apresentados. Deverão ser sempre considerados, dentro do critério de avaliação da incapacidade, a possibilidade de agravamento da doença e o risco de vida para a pessoa ou para terceiros, que a continuação do trabalho possa acarretar, sob determinadas condições em que ele é executado pelo servidor. $\mathrm{O}$ conceito de incapacidade deve ser analisado quanto ao grau, à duração e à profissão ou função desempenhada. Entende-se por invalidez a incapacidade laborativa total, permanente e omniprofissional, insuscetível de recuperação ou readaptação profissional, em consequência de doença ou acidente $^{14}$

O acometimento seroso no LES está associado à fase aguda da doença, quando as alterações podem ser detectadas clínica e subsidiariamente. $\mathrm{Na}$ fase de exacerbação do lúpus, é comum o encontro de pequenos derrames pericárdicos, podendo, entretanto, um grande derrame pericárdico ser a primeira manifestação da doença. A pleurisia lúpica ocorre em $50 \%$ dos pacientes com a doença em atividade, ocasionalmente com derrame pleural bilateral ${ }^{6,8}$.

A pericardite lúpica e a pleurisia podem apresentar-se de forma leve, quando a doença se encontra estável, não há comprometimento das funções vitais, sem repercussão funcional significativa, caracterizando a doença oligossintomática, em que as doses baixas dos medicamentos, caso estejam em uso, não desencadeiam sintomas secundários significativos. A serosite lúpica nos graus moderado a severo, com sinais e sintomas gerais e específicos exuberantes, associados a alterações laboratoriais significativas, que podem requerer tratamento em regime de internação hospitalar com prescrição de elevadas doses de corticosteróides e imunossupressores, produz significativa gama de efeitos medicamentosos secundários que se somam aos sinais e sintomas da doença, condição que determina incapacidade.

A evolução clínica da doença varia de caso a caso e não é possível estabelecer um período para o tratamento da doença ativa ou prever quando entrará em remissão. A doença exige acompanhamento clínicolaboratorial, com observação sequencial até a remissão. Com a melhora clínica, as doses dos medicamentos são reduzidas progressivamente, podendo permanecer em doses baixas de manutenção durante longo período. Desta forma, no lapso em que o paciente apresentar a doença ativa, considerando também os efeitos secundários dos medicamentos, é possível caracterizar quadro de incapacidade laborativa.

Nos casos de evolução para estado oligossintomático, com baixas doses medicamentosas de manutenção, com regressão dos sinais e sintomas, o paciente poderá ser liberado para o trabalho. O LES pode evoluir com lesões permanentes ou sequelas irreversíveis não só em decorrência da própria doença como em consequência do tratamento medicamentoso. A utilização do SLICC/ACR/DI (quadro 2) apresenta grande ferramenta para a quantificação das sequelas e doenças. Entretanto, a decisão quanto à capacidade laborativa vai depender da avaliação do quadro clínico individualizado, na qual será levado em conta o grau de comprometimento funcional.

Para avaliação da incapacidade laborativa, é importante identificar os sinais e sintomas que determinam limitação funcional significativa. O LES em atividade produz sintomas gerais como intenso cansaço, fadiga e febre. No caso do derrame pleural, elenca-se a dor torácica do tipo pleurítica, que é o sintoma mais comum no derrame pleural, indica comprometimento da pleura parietal, e é mais intensa na fase inicial da pleurite, melhorando com o aumento do derrame pleural. A dor é intensa, geralmente descrita em pontadas, que piora com a inspiração profunda e com a tosse, melhorando com o repouso do lado afetado, localizada na área pleural afetada. A tosse seca pode estar associada. A dispneia estará presente especialmente nos derrames mais volumosos e nos de rápida formação, de observação objetiva com esforço respiratório, frequência respiratória acima de 20 incursões por minuto, fadiga dos músculos respiratórios, hipoxemia com saturação de oxigênio abaixo de 90\%, hipercapnia, acidose, confusão mental, palidez cutânea, sudorese fria, cianose, taquipnéia, utilização de musculatura acessória e tiragem intercostal. A ausculta mostra redução ou abolição do murmúrio vesicular, o qual pode estar aumentado na borda superior do derrame pleural em seu limite com o parênquima pulmonar. Há redução ou ausência do frêmito tóracovocal e a percussão mostra macicez ou submacicez na região com líquido. Nos derrames volumosos, pode ser observado abaulamento do hemitórax acometido ${ }^{15,16}$.

A pericardite aguda decorre da inflamação do pericárdio causando dor torácica subesternal agravada pelos movimentos respiratórios, ao tossir ou ao curvar-se para frente, dispneia, febre, atrito pericárdico, taquicardia e alterações eletrocardiográficas com ondas T apiculadas e elevação do segmento ST, semelhante a outras causas de pericardite. $\mathrm{O}$ ecocardiograma é considerado o método de escolha para avaliação do derrame pericárdico, 
entretanto, a ausência de efusões pericárdicas à ecocardiografia não exclui a possibilidade de pericardite suspeita clinicamente. A efusão pericárdica pode ocorrer em pequena quantidade, sem expressão clínica ou evoluir para grandes volumes compressivos, caracterizando tamponamento cardíaco ${ }^{17,18}$.

O LES pode entrar em fase de exacerbação caracterizada pela serosite lúpica, que pode englobar a pleurisia e a pericardite. Os sinais e sintomas da serosite acima descritos determinam limitação funcional que pode ser de leve a grave, de acordo com o comprometimento da doença. A avaliação da incapacidade deve levar em conta os sinais e sintomas gerais e específicos da doença, o tratamento medicamentoso vigente e os efeitos colaterais dos medicamentos utilizados. Em geral, a apresentação da doença é na forma branda e responsiva ao tratamento medicamentoso, com bom prognóstico $^{19}$. A serosite lúpica, desta forma, pode determinar incapacidade temporária para o trabalho, requerendo avaliações periódicas até a constatação da doença oligossintomática ou em remissão, na qual os sinais e sintomas regrediram.

\section{Conclusão}

Os sinais e sintomas da serosite do LES que podem mostrar a incapacidade são dispneia, tosse, dor torácica do tipo pleurítica, dor torácica subesternal, dor torácica que se agrava com a movimentação respiratória, utilização da musculatura acessória, atrito pleural, atrito pericárdico, murmúrio vesicular diminuído ou abolido, hipoxemia e taquicardia.

Leva-se também em conta que o tratamento medicamentoso instituído, cujos efeitos secundários exuberantes nas doses elevadas incluem a possibilidade de complicações infecciosas pela imunossupressão, indica que a avaliação quanto à incapacidade deve ser realizada caso a caso.

Considerando que a serosite lúpica apresenta evolução clínica favorável mediante tratamento medicamentoso, com bom prognóstico na maioria dos casos, é possível concluir que a incapacidade decorrente da doença ativa é em geral total e temporária, exigindo reavaliações periódicas com o objetivo de verificação clínico-laboratorial da evolução da doença.

Chammas NSR; Cintra RB. Medical expert evaluation of work capacity of patients with lupus serositis. Saúde, Ética \& Justiça. 2014;19(1);12-20.

\begin{abstract}
Systemic lupus erythematosus (SLE) is a chronic inflammatory disease of autoimmune character and of unknown etiology that predominantly affects females at a frequency of 9:1 and it can reach various organs and physiological systems, most commonly the skin, joints and kidneys. However, lupus serositis has a significant impact on patients, with around $50 \%$ of cases having pericardial and pleural involvement whose clinical manifestations can result in an incapacity to work. The objective of this study was to determine when lupus serositis leads to disability, resulting in the granting of social security benefits. For this, the authors did a literature review on the subject, in order to evaluate the work capability of patients with lupus serositis. The lupus activity may result in the incapacity to work. Most people with SLE may have a normal productive life; however, some patients have a more complicated course and are no longer able to work. Some signs and symptoms of lupus serositis can show evidence of the disability. It is possible to conclude that a disability resulting from acute disease is usually total and temporary, requiring periodical reevaluations aiming for clinical and laboratorial control of disease progression.
\end{abstract}

KEYWORDS: Serositis; Lupus erythematosus, Systemic; Work capacity evaluation.

\title{
Referências
}

1. Borba EF, Latorre LC, Brenol JCT, Kayser C, Silva NA, Zimmermann AF, et al. Consenso de Lúpus Eritematoso sistêmico, Rev Bras Reumatol. 2008; 48(4):196-207. DOI: http://dx. doi.org/10.1590/S0482-50042008000400002.

2. OMS - Organização Mundial da Saúde. Classificação Estatística Internacional de Doenças e Problemas Relacionados à Saúde. Décima revisão. São Paulo: Centro Colaborador da OMS para Classificação de Doenças em Português; 1993.

3. Appenzeller S, Costallat LTL. Análise de sobrevida global e fatores de risco para óbito em 509 pacientes com lúpus eritematoso sistêmico (LES). Rev Bras Reumatol. 2004; 44(3):198-205. DOI: http://dx.doi.org/10.1590/S0482-

\section{4.}

4. Sato EI, Lupus Eritematoso Sistêmico. In: Borges DR, Rothschild HA, editores. Atualização Terapêutica. Porto Alegre: Artes Médicas; 2005. p.1720-6.

5. Brasil. Ministério da Previdência Social. Anuário Estatístico da Previdência Social. Brasília (DF): DATAPREV; 2012.

6. Ferreira FN, Martins JR, Riso MN, Soromenho FC, Riscado MV, Baptista LMM. Lupus Eritematoso Disseminado. Revisão de 20 casos. Acta Médica Portuguesa. 1988; 1:17-28.

7. Brasil. Ministério da Saúde. Portaria $n^{0} 100$ de 07 de fevereiro de 2013. Aprova o Protocolo Clínico e Diretrizes 
Terapêuticas do Lúpus Eritematoso Sistêmico. [Internet] Diário Oficial da União, Brasília (DF). 201308 fev. [acesso em 10 dez 2013]. Disponível em: http://bvsms.saude.gov. br/bvs/saudelegis/sas/2013/prt0100_07_02_2013.html

8. Magalhães MB, Donadi EA, Louzada Jr P. Manifestações Clínicas do Lúpus Eritematoso Sistêmico: Abordagem Diagnóstica e Terapêutica na sala de urgência. Simpósio: Urgências e Emergências Imunológicas. 2003; 36:409-17.

9. Alves LJ, Hydalgo L, Rolim LF, Campagnone GZ, Aidar MT, Novaes GS, et al. Avaliação Clínica e Laboratorial da Cardiopatia no Lúpus Eritematoso Sistêmico. Arq Bras Cardiol.1997; 68(2):79-83.

10. Chile. Ministerio de Salud. Guía Clínica AUGE Lupus Eritematoso Sistémico. Santiago: Minsal, 2013.

11. Sato El, Bonfá ED, Costallat LTL, Silva NA, Brenol JCT, Santiago MB, et al. Lúpus Eritematoso Sistêmico: Tratamento do Acometimento Sistêmico. Rev Bras Reumatol. 2004; 44(6):458-63. DOI: http://dx.doi.org/10.1590/ S0482-50042004000600010.

12. Freire EAM, Souto LM, Ciconelli RM. Medidas de avaliação em lúpus eritematoso sistêmico. Rev Bras Reumatol. 2011; 51(1):75-80. DOI: http://dx.doi.org/10.1590/S048250042011000100006 .

13. Guarize J, Apénzeller S, Costallat LTL. Avaliação do índice de danos permanentes através do SLICC/ACR-DI em pacientes brasileiros com lúpus eritematoso sistêmico. Rev Bras Reumatol. 2004; 44(2):109-14. DOI: http://dx.doi. org/10.1590/S0482-50042004000200002.

14. Previdência Social. Instituto Nacional do Seguro Social. Manual de Perícia Médica da Previdência Social. [Internet] [acesso em $13 \mathrm{dez}$ 2013] Disponível em http://www.ieprev. com.br/userfiles/file/tabela\%20de $\% 20$ teto\%20inss/manualdepericiasmedicasdoINSS.pdf

15. Ribeirão Preto. Serviço de Atendimento Médico de Urgência. Dispneia e Insuficiência Respiratória. 2012.

16. Pinheiro BV, Oliveira JCA, Jardim JR. Derrame Pleural. Pneumo Atual [Internet]. 2000 Out. Disponível em http://www.pneumoatual.com.br/secao/per/per. aspx?idAssunto $=189$.

17. Zanettini MT, Zanettini JO, Zanettini JP. Pericardite. Série de 84 casos consecutivos. Arq Bras Cardiol. 2004; 82(4):360-4. DOI: http://dx.doi.org/10.1590/S0066$-782 \times 2004000400007$.

18. Falcão CA, Lucena N, Alves IC, Pessoa AL, Godói ET. Lupus Carditis. Arq Bras Cardiol. 2000; 74(1):64-71. DOI: http://dx.doi.org/10.1590/S0066782X2000000100007.

19. Brasil. Ministério da Previdência Social. Instituto Nacional do Seguro Social. Diretrizes de apoio à decisão médico pericial em Clínica Médica. Parte I. [Internet] Brasília, DF; 2009. Disponível em: http://www.previdenciasocial.gov.br/ arquivos/office/4_091021-153135-494.pdf 